\title{
Long-duration simulations of the evolution of jet-driven molecular outflows
}

\author{
R. Keegan and T. P. Downes
}

\author{
School of Mathematical Sciences, Dublin City University, Glasnevin, Dublin 9, Ireland \\ e-mail: turlough.downes@dcu.ie
}

Received 26 October 2004 / Accepted 22 March 2005

\begin{abstract}
We use numerical simulations to examine the mass-velocity, $m(v)$, and intensity-velocity, $I(v)$, relations in jet-driven molecular outflows up to an age of $2300 \mathrm{yr}$. We find that the $m(v)$ relation is a power-law, $m(v) \propto v^{-\gamma}$, the exponent of which increases with time up to $\gamma \approx 1.6$ at $t \approx 1500 \mathrm{yr}$. It then becomes roughly constant. This indicates that $\gamma$ does not evolve throughout the lifetime of a molecular outflow, at least in the context of the jet-driven model.

We also investigate the effect of long-period episodicity of the jet on the $m(v)$ and $I(v)$ relations. We find that, contrary to previous expectations, these relations are not significantly changed with the introduction of such variability into the jet.

Finally, we present a novel, and relatively simple, parallelisation method for the code used in these simulations. This gives an increase of roughly a factor of 4 in speed over standard methods, and allows the simulations presented here to be run fairly easily, even with modest computational resources.
\end{abstract}

Key words. hydrodynamics - shock waves - ISM: jets and outflows - ISM: molecules

\section{Introduction}

Various authors have noted that the intensity-velocity relationship observed in low-J CO lines in molecular outflows tends to follow a broken power-law $I_{\mathrm{CO}}(v) \propto v^{-\gamma}$ with $\gamma \approx 1.5-2$ up to line-of-sight velocities $v_{\text {break }} \approx 10-30 \mathrm{~km} \mathrm{~s}^{-1}$ and $\gamma \approx 3-7$ at higher velocities (e.g. Rodríguez et al. 1982; Stahler 1994; Bachiller \& Tafalla 1999; Richer et al. 2000). More recently, similar behaviour has been observed in observations of the $\mathrm{H}_{2} \mathrm{~S}(1)$ 1-0 line in various outflows (Salas \& Crúz-González 2002). The exponents in this latter intensity-velocity relation, $I_{\mathrm{H}_{2}}(v)$ tend to be smaller than those in the $I_{\mathrm{CO}}(v)$ relations for the same outflows. These authors also suggested that $v_{\text {break }}$ may be lower for $I_{\mathrm{H}_{2}}(v)$ than for $I_{\mathrm{CO}}(v)$ but it is difficult to be certain of this due to the relatively low spectral resolution of their observations $\left(\sim 25 \mathrm{~km} \mathrm{~s}^{-1}\right)$.

The $I_{\mathrm{CO}}(v)$ relation has been reproduced in numerical simulations of jet-driven outflows where the molecular outflow is identified as the molecular material which has been swept up by the bowshock created by the jet advancing into the ambient medium (e.g. Smith et al. 1997; Downes \& Ray 1999; Downes $\&$ Cabrit 2003). In addition, Downes \& Cabrit (2003) reproduced qualitatively similar $I_{\mathrm{H}_{2}}(v)$ relations to those observed by Salas \& Crúz-González (2002). Rosen \& Smith (2004) have also reported on $I_{\mathrm{H}_{2}}(v)$ profiles calculated from their simulations. These latter relations appear to be somewhat different to those observed, particularly at low velocities. It is likely that at least some of the difference can be attributed to problems with the resolution of these latter simulations and this will be the subject of a forthcoming paper.

All these numerical simulations show a systematic increase with time of $\gamma$ at low velocities. This behaviour of $\gamma$ appears to be supported by the observations (e.g. Richer et al. 2000 and references therein; Salas \& Crúz-González 2002). To date, simulations have only addressed the early-time behaviour of these outflows, with simulation ages being typically in the range of 400-1000 yr. This leaves open the question of whether the temporal behaviour of $\gamma$ seen in simulations is something which only occurs in young jet-driven flows or, indeed, whether it is a transient effect arising from initial conditions in the simulations.

Downes \& Ray (1999) found no evidence that variability in the jet velocity affects the value of $\gamma$, even for large variations (up to $60 \%$ ) of the jet velocity. However, the variations were of short period (only up to $50 \mathrm{yr}$ ) and these authors suggested that large variations over much longer periods may have a significant impact on $\gamma$. They were unable to examine this possibility due to computational constraints restricting the age to which they could simulate jets, and hence the time-scales associated with the variability of the jet. Moreover, Arcé \& Goodman (2001) used an analytic treatment of the problem of episodic outflows to suggest that episodicity could, in fact, increase the value of $\gamma$.

In this paper we present the results of two long-duration simulations of jet-driven molecular outflows up to ages 
of $2300 \mathrm{yr}$, one of which is for an episodic jet with a periodicity of $800 \mathrm{yr}$. The overall aim is to assess the long term evolution of jet-driven molecular outflows: in particular we address whether $\gamma$ evolves to a steady state and also the effect of relatively long-period episodicity on the predicted observational characteristics of such flows.

The structure of this paper is as follows. In Sect. 2 we present some details of the code used in this work, along with the method of parallelisation and the initial conditions used for the simulations. In Sect. 3 we discuss the results of the simulations in the context of the points raised above and, finally, in Sect. 4 we summarise our results and their significance for jet-driven outflows.

\section{Numerical model}

The code used in this work is an updated version of that described in Downes \& Ray (1998). It is a hydrodynamical code and the simulations are performed in 2D cylindrical symmetry. To achieve the long time scales we require for this work we have developed a novel approach for parallelising the code for execution on a distributed memory cluster. We begin by giving some details of the equations and numerical method, followed by details of the method of parallelisation, and finally we detail the initial conditions used in the simulations.

\subsection{Equations and numerical method}

The equations solved are

$\frac{\partial \rho}{\partial t}=-\nabla \cdot(\rho \boldsymbol{u})$

$\frac{\partial(\rho \boldsymbol{u})}{\partial t}=-\nabla \cdot[\rho \boldsymbol{u} \boldsymbol{u}+p \boldsymbol{I}]$

$\frac{\partial e}{\partial t}=-\nabla \cdot[(e+p) \boldsymbol{u}]-L$

$\frac{\partial n_{\mathrm{H}} x}{\partial t}=-\nabla \cdot\left[n_{\mathrm{H}} x \boldsymbol{u}\right]+J\left(x, n_{\mathrm{H}}, T\right)$

$\frac{\partial n_{\mathrm{H}_{2}}}{\partial t}=-\nabla \cdot\left[n_{\mathrm{H}_{2}} \boldsymbol{u}\right]-n_{\mathrm{H}_{2}} n_{\mathrm{H}} k(T)$

$\frac{\partial n_{\mathrm{H}}}{\partial t}=-\nabla \cdot\left[n_{\mathrm{H}} \boldsymbol{u}\right]+2 n_{\mathrm{H}_{2}} n_{\mathrm{H}} k(T)$

$\frac{\partial \rho \tau}{\partial t}=-\nabla \cdot(\rho \tau \boldsymbol{u})$

where $\rho, \boldsymbol{u}, p, I$ and $e$ are the mass density, velocity, pressure, identity matrix and total energy density respectively. $n_{\mathrm{H}}$ is the number density of atomic hydrogen and $n_{\mathrm{H}_{2}}$ is the number density of molecular hydrogen. $x$ is the ionisation fraction of atomic hydrogen, $T$ is the temperature, $k(T)$ is the dissociation coefficient of molecular hydrogen, $J\left(x, n_{\mathrm{H}}, T\right)$, is the rate of ionisation or dissociation of atomic hydrogen and $\tau$ is a scalar which is used to track the jet gas. We also have the following definitions

$p=n k T$

$$
\begin{aligned}
& e=\frac{1}{2} \rho \boldsymbol{u} \cdot \boldsymbol{u}+\frac{c_{\mathrm{v}}}{k_{\mathrm{B}}} p \\
& L=L_{\mathrm{rad}}+E_{\mathrm{I}} J\left(x, n_{\mathrm{H}}, T\right)+E_{\mathrm{D}} k(T)
\end{aligned}
$$

where $c_{\mathrm{V}}$ is the specific heat at constant volume, $k_{\mathrm{B}}$ is Boltzmann's constant, $E_{\mathrm{I}}$ is the ionization energy of hydrogen and $E_{\mathrm{D}}$ is the dissociation energy of $\mathrm{H}_{2} . L$ represents the energy loss and gain due to radiative and chemical processes. $L_{\mathrm{rad}}$ is the loss due to radiative transitions and consists of a function for losses due to atomic transitions (Sutherland \& Dopita 1993), and one for losses due to molecular transitions (Lepp \& Shull 1983). The second and third term in (10) represent the energy dumped into the ionisation of $\mathrm{H}$ and the energy dumped into dissociation of $\mathrm{H}_{2}$ respectively. The dissociation coefficient $k(T)$ comes from Dove \& Mandy (1986) and the ionization rate, $J$, is that used by Falle \& Raga (1995). Note that we do not include $\mathrm{H}_{2}$ reformation. We calculated a posteriori the maximum possible $\mathrm{H}_{2}$ reformation rate based on the maximum density attained in our simulations and found that the associated timescale was of order the duration of the simulation itself. Hence we can conclude that reformation is not significant for the simulations presented here.

To solve the equations on the $2 \mathrm{D}$ grid we use a temporally and spatially second order accurate MUSCL scheme (van Leer 1977). The fluxes at the cell boundaries are determined using a linear Riemann solver except where the resolved pressures on either side of the cell boundary differ by more than $10 \%$. In this case a non-linear Riemann solver is used. Adopting this strategy allows us to benefit from the computational efficiency of the linear solver while allowing for correct treatment of strong rarefactions with the non-linear solver.

\subsection{Parallelisation method}

The usual approach to parallelising these types of codes is to split the computational domain between available processors such that all will have approximately the same number of computations to perform. In the case of jet simulations, however, we do not utilise the computational resources efficiently, as many processors are integrating the relevant equations for quiescent parts of the grid for much of the simulation. To better focus the computational resources we have developed a more sophisticated algorithm for sharing out portions of the dynamically varying part of the grid among all the processors.

Each processor integrates a small portion of this part of the grid and, as the outflow increases in size, the size of this portion increases accordingly. To achieve this, it is necessary to redistribute cells between the processors throughout the simulation in order to maintain the overall continuity of the outflow. Figure 1 illustrates how the method works for a simulations of an adiabatic jet using 8 processors.

One of the possible bottle-necks in this type of algorithm occurs in the redistribution of the cells between the processors. However, it was found that this added overhead was negligible relative to the overall time for the simulation. Figure 2 shows a comparison between the simple and redistributing parallelisation methods, in terms of speedup, utilising up to 28 processors. 

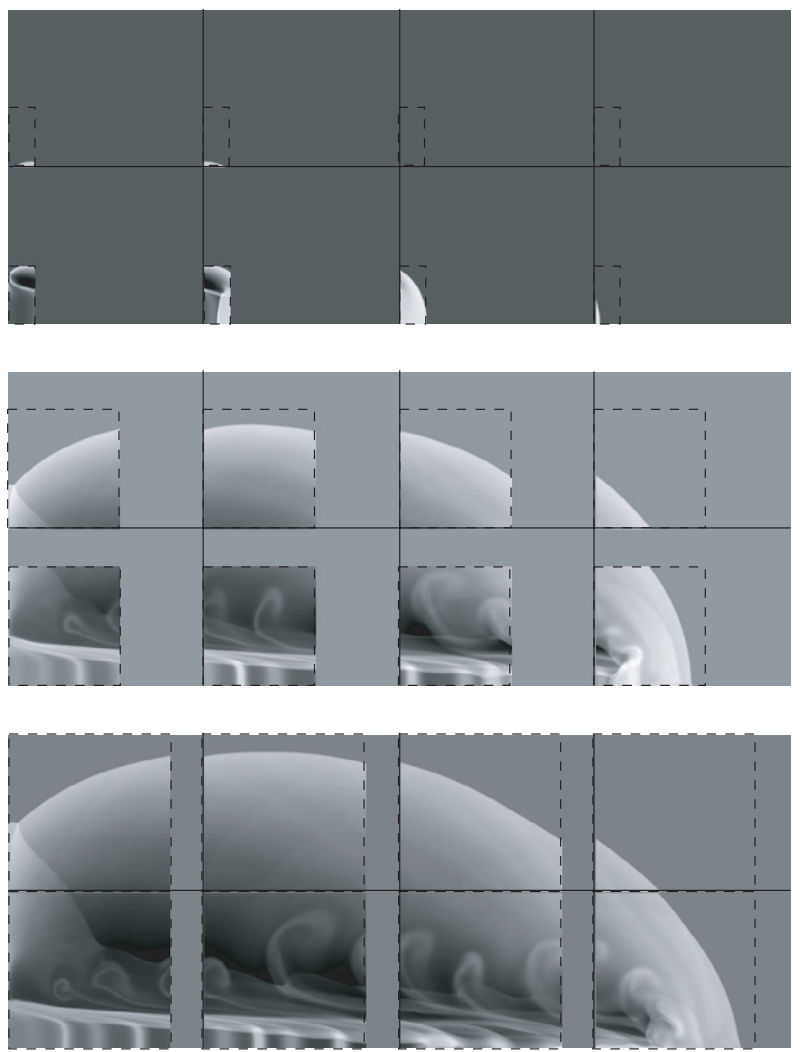

Fig. 1. An example of an adiabatic jet simulation run over eight processors, the domains of which are indicated by the solid lines. Each image is taken at different stages in the evolution of the flow. As the jet propagates further into the computational grid, each process computes over a larger and larger area (shown by the dashed-line boxes).

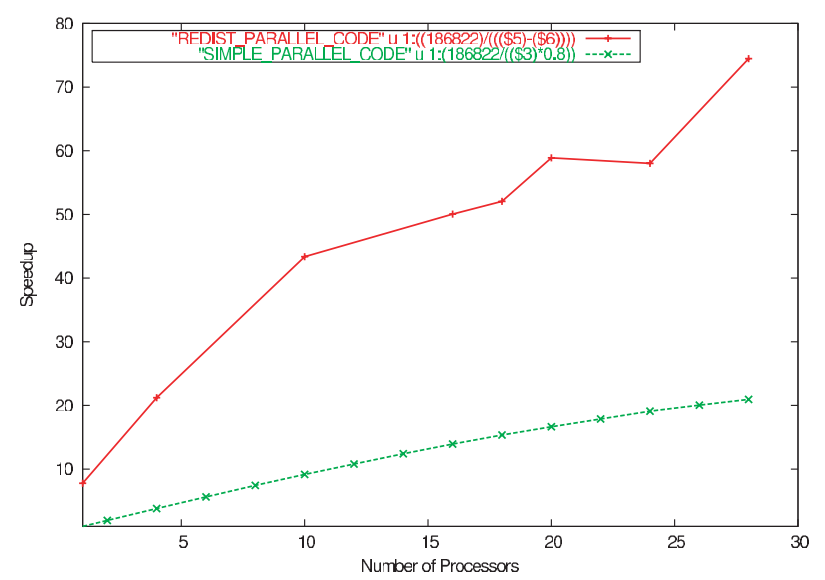

Fig. 2. Comparison between simple and redistributing parallel codes for an adiabatic jet simulation over 10000 time-steps.

The speedup is given relative to using a single processor computing over the entire grid for 10000 time-steps. It can be seen that our method of parallelisation gives a speed-up of nearly a factor of 4 over the standard parallelisation method. This is what would be expected for a $2 \mathrm{D}$ simulation if the rate of expansion of the dynamically varying region of the grid is constant with time.

\subsection{Initial conditions}

The initial conditions used here are the same as those used in Downes \& Cabrit (2003), but we detail them here for completeness. The computational domain consists of a $10^{4}$ by $10^{3}$ (or $0.32 \mathrm{pc} \times 0.032 \mathrm{pc}$ ) grid. The boundary conditions are set to be reflecting on $r=0$ (the jet axis) and $z=0$ except where the jet enters the grid. On all other boundaries the gradient is set to zero.

Both of the simulations presented in this paper are of jets which are density matched with the ambient medium at a value of $100 \mathrm{~cm}^{-3}$. This density is somewhat low in comparison to observations of these systems. However, this low density is necessary as it allows us to resolve the post-shock cooling lengths, at least behind the stronger shocks in the simulation where the most dynamically significant evolution takes place. It also means that we calculate the momentum transfer correctly (Downes \& Ray 1999). In order to maintain this resolution of the post-shock cooling regions, the spatial resolution (here $1 \times 10^{14} \mathrm{~cm}$ ) should be scaled as the inverse square of the density. Thus simulating higher densities becomes impractical very quickly.

The ambient temperature is set to $10^{2} \mathrm{~K}$ and the jet temperature to $10^{3} \mathrm{~K}$. The ambient temperature is a little higher than that expected for molecular clouds and we choose this for numerical reasons. However, this choice does not affect the dynamics or observational characteristics of the post-shock ambient gas, which is what we are interested in for the purposes of this work. The jet temperature seems reasonable from observations of jets with molecular emission (e.g. Noriega-Crespo et al. 1995).

The energy loss/gain function $L$ is set to zero below $10^{3} \mathrm{~K}$ as the data used in the cooling functions is unreliable below this temperature and cooling is not dynamically significant here either. The ratio of the number density of molecular hydrogen to that of atomic hydrogen is set to be $n_{\mathrm{H}_{2}} / n_{\mathrm{H}}=9$ within the jet and in the ambient medium. The gas is assumed to be one of solar abundances.

In this paper we present results for two different jets: a jet with a periodic input velocity and one with an "episodic" input velocity. Each jet enters the grid at $z=0$ and $r \leq R$. In all cases $R$ was set to be 50 cells $\left(5 \times 10^{15} \mathrm{~cm}\right)$. For the periodic jet the input jet velocity was set to have a time-averaged velocity of $v_{0}=215 \mathrm{~km} \mathrm{~s}^{-1}$. Sinusoidal variations are superimposed upon the input velocity with periods of 5, 10, 20 and $50 \mathrm{yr}$ with a total amplitude of $v_{1}$ with the ratio $v_{1} / v_{0}=0.16$ for the periodic jet.

For the episodic jet we have set $v_{1} / v_{0}=0\left(v_{0}=\right.$ $215 \mathrm{~km} \mathrm{~s}^{-1}$ ). However, in this case, the velocity was set to $v_{0}$ for $400 \mathrm{yr}$, and then to a low velocity $\left(30 \mathrm{~km} \mathrm{~s}^{-1}\right)$ for $400 \mathrm{yr}$, thus representing episodic ejections with period 800 yr. Rather than switching the jet between these two behaviours instantaneously, the startup and termination of each pulse is ramped. The value for the velocity varies linearly from $30 \mathrm{~km} \mathrm{~s}^{-1}$ to $v_{0}$ over the course of 50 years at the beginning of the pulse and inversely at the end of the pulse. This type of behaviour has been suggested (Lim et al. 2002) to give rise to increased molecular 


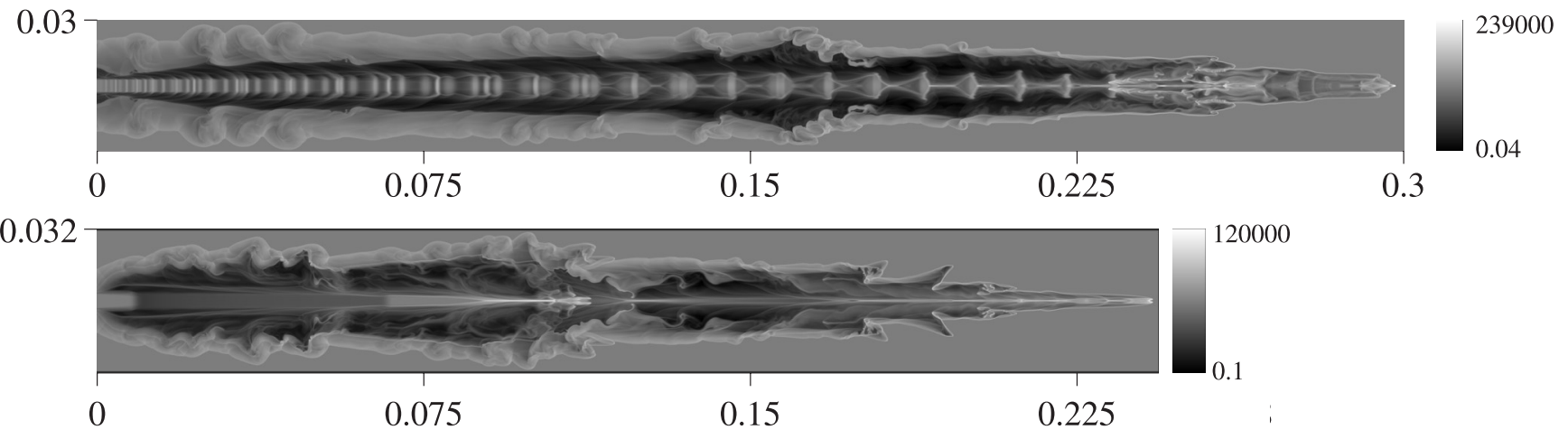

Fig. 3. Log-scale plot of the number density distribution for the pulsed jet (top) and episodic jet (bottom) simulations at $t=2300$ yr. The grey-scale density units are in $\mathrm{cm}^{-3}$ and the length-scale is in parsecs.

abundances at the head of the bowshock, which in turn, may affect the intensity-velocity relation.

To prevent spurious numerical waves being emitted from the edge of the jet as it enters the computational domain we have inserted a shear layer of about 5 cells. The values for the density, pressure and velocity in these cells are given by a linear profile joining the jet conditions with those prevailing in the cell just outside the jet boundary at the $z=0$ axis. The actual form of this shear layer is relatively unimportant in this work as the jet does not entrain molecular material via this shear layer due to the low density of the surrounding cocoon (e.g. Blondin et al. 1990) and the low growth rates of the Kelvin-Helmholtz instability in these high Mach number jets (e.g. Rossi et al. 1997).

Finally, we set the jet tracer variable, $\tau$, to be 1 in the boundaries from which the jet flows and zero elsewhere. This means that $\tau$ gives us the fraction of material in any cell which originated in the jet.

\section{Results}

In this section we present the results of the two simulations. We start off by discussing the results of the pulsed jet. We then go on to discuss the simulation of the episodic jet in the context of the results from the pulsed jet simulation.

\subsection{Morphology}

It can be seen from Fig. 3 that the molecular outflow, identified as the material in the bowshock of the jet is rather irregular, with "corrugations" along its length. These corrugations have been noted before in other simulations (e.g. Smith et al. 1997; Downes \& Ray 1999) and are the result of a fluid instability at the head of the bowshock, most likely the Vishniac instability (Dgani et al. 1996).

The length-to-width ratio of the molecular outflow produced in this simulation is approximately 10.2. This rather large ratio has been noted before by several authors (Lee et al. 2000 and references therein) and suggests that precession, or imperfect collimation would be necessary in order to explain at least some outflows (e.g. RNO43, Bence et al. 1996), although others may be directly accounted for without these properties (e.g. HH211, Richer et al. 2000).

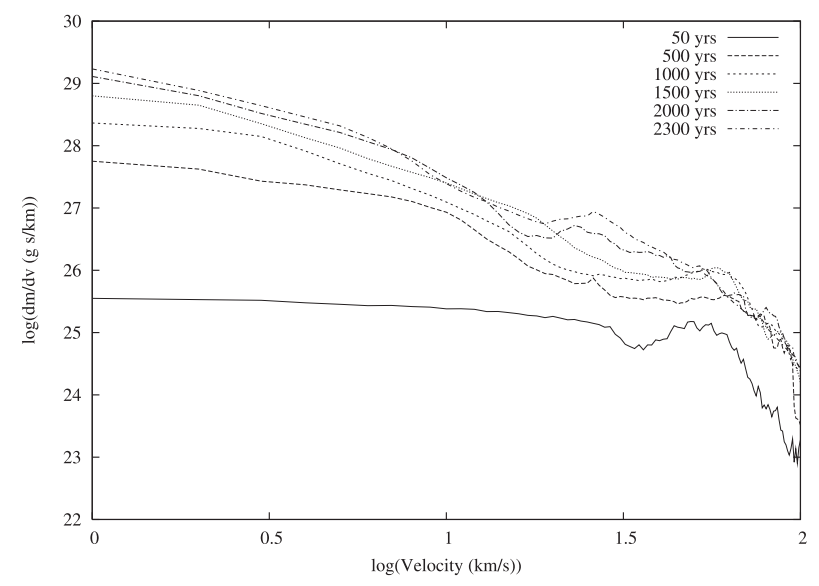

Fig. 4. Plots of the total swept-up mass versus velocity, $m(v)$, for different times for the pulsed jet simulation.

\subsection{The mass-velocity relation}

The mass-velocity and intensity-velocity relations are presented for swept-up material (i.e. neglecting all material which was initially injected in the jet). We do this because the jetdriven model postulates that it is the shocked ambient material, rather than jet material, which constitutes the molecular outflow (e.g. Raga \& Cabrit 1993). We first split the azimuthal direction in the simulation (considered to be ignorable in the simulations) into 100 zones. We then calculate the line-of-sight velocity to the observer in each of these zones for each value of $z$ and $r$ in the simulation. This velocity determines into which velocity bin the relevant quantity (mass, $\mathrm{CO}$ intensity etc) will be added. In this work we use a bin width of $1 \mathrm{~km} \mathrm{~s}^{-1}$. We use the jet tracer variable, $\tau$, to ensure we only add in these quantities for the ambient material. In this way we obtain the predicted differential mass-velocity and intensity-velocity relationships.

Figure 4 shows plots of the total swept-up mass-velocity relation, $m(v)$, at different times for the pulsed jet simulation. At low velocities $\left(v \leq 20 \mathrm{~km} \mathrm{~s}^{-1}\right)$ the relation settles to a steady state at around $t=1500 \mathrm{yr}$. It is worth noting that, even for late times, there continue to be substantial variations in $m(v)$ for $v>20 \mathrm{~km} \mathrm{~s}^{-1}$. These variations result from the corrugations along the bowshock discussed in Sect. 3.1. These irregularities 


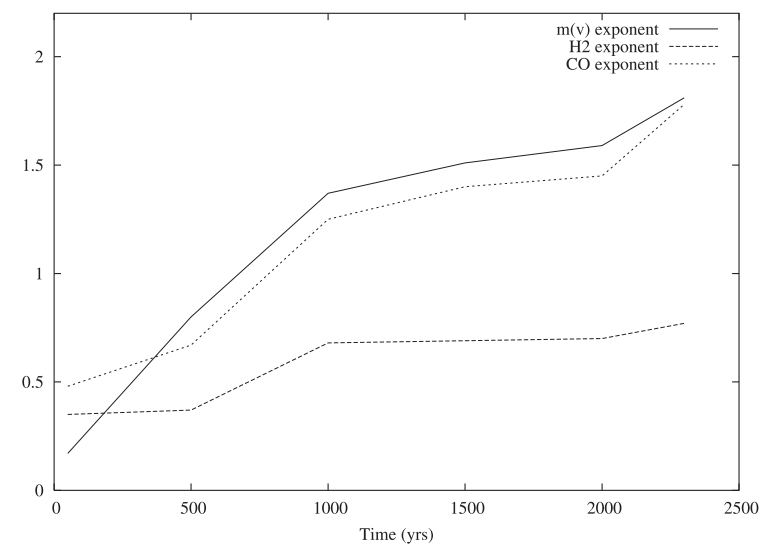

Fig. 5. Plots of the exponents in the $m(v), I_{\mathrm{H}_{2}}(v)$ and $I_{\mathrm{CO}}(v)$ relations, as a function of time. They are calculated by fitting over the velocity range $1-10 \mathrm{~km} \mathrm{~s}^{-1}$.

contain a large amount of material and so appear as "bumps" on the $m(v)$ relation. Observed over time these bumps can be seen to appear at high velocity and gradually move to lower velocity before disappearing into the power-law part of the $m(v)$ relation below $v \approx 20 \mathrm{~km} \mathrm{~s}^{-1}$.

As mentioned in Sect. 1, one of the important questions raised by previous numerical simulations of jet-driven molecular outflows is whether the exponent, $\gamma$, in the $m(v)$ relation will reach a steady state, given that at early times it increases. We can see from Fig. 5 that, for $t<1500 \mathrm{yr}$, the exponent of the mass-velocity relation increases monotonically with time. This increase is postulated to result from the bowshock creating a large reservoir of slowly moving material as it ages and moves into the computational domain (Downes \& Cabrit 2003). Smith et al. (1997) suggested that the exponent increase is a result of the bowshock changing shape with time. This, however, seems unlikely given our results shown in Fig. 7 (see later in this section). However, for $t>1500$ yr $\gamma$ remains approximately constant until $1800 \mathrm{yr}$ before beginning to increase slightly again.

A careful examination of the reason for the increase in $\gamma$ at later times leads us to believe that it results from the large variations at higher velocities propagating into this velocity range (cf. Fig. 4), unlike the process of general steepening seen at earlier times.

It is also clear from Fig. 5 that the slopes of the $I_{\mathrm{H}_{2}}(v)$ and $I_{\mathrm{CO}}(v)$ relations increase with time, but stabilise at about the same time as the $m(v)$ relation. We can also see that, as expected, the $I_{\mathrm{H}_{2}}(v)$ relation is shallower than either of the other two relations (see Sect. 3.3).

In order to allow inspection of the overall behaviour of $m(v)$ for $t>1500 \mathrm{yr}$ we present plots of of $m(v)$ and $m_{\mathrm{H}_{2}}(v)$ timeaveraged over $t \in[1500,2300]$ yr in Fig. 6. An important thing to note about these plots is that there is no obvious break in the slope of $m(v)$. This has been noted before by Downes \& Cabrit (2003) for shorter duration simulations, and is at odds with the assumption, made by many authors (e.g. Zhang \& Zheng 1997), that $m(v)$ should follow a broken power-law. It can also be seen from Fig. 6 that $m_{\mathrm{H}_{2}}(v)$ is considerably steeper than $m(v)$ for $v>20 \mathrm{~km} \mathrm{~s}^{-1}$. This was also found by Downes \& Cabrit (2003) for their shorter-duration simulations and can be

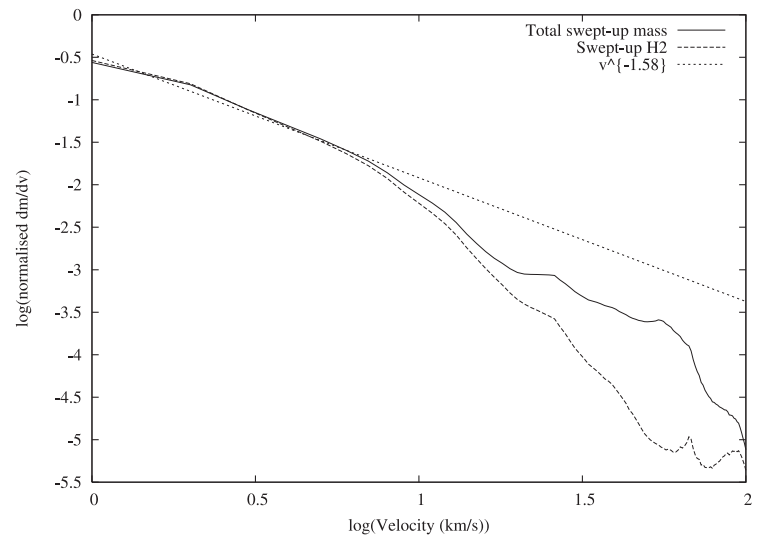

Fig. 6. Plots of the $m(v)$ and $m_{\mathrm{H}_{2}}(v)$ relations, time-averaged over $t=$ $1500 \mathrm{yr}$ to $t=2300 \mathrm{yr}$ and normalised by the total of each variable integrated over velocity. Shown for reference is the relation $v^{-1.58}$, the best fit power-law to the low-velocity behaviour of $m(v)$.

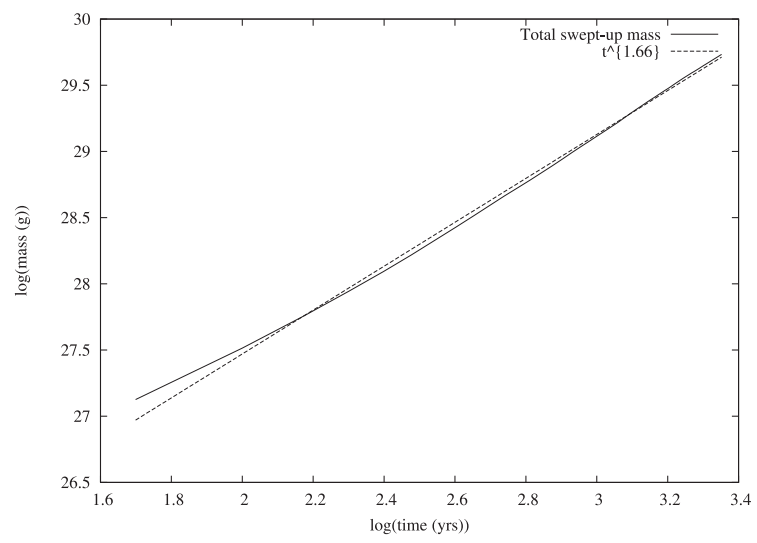

Fig. 7. Plot of the total swept-up mass as a function of time, along with the best-fit power-law, which has an exponent of 1.66 .

explained by noting that material at higher velocities has gone through stronger shocks and hence one expects higher levels of dissociation of $\mathrm{H}_{2}$.

Finally, Fig. 7 contains a plot of the total swept-up mass as a function of time. Downes \& Cabrit (2003) have argued that, if the bowshock can be assumed to be a power-law with $z \propto r^{s}$ then $m(t) \propto t^{\beta}$ and $m(v) \propto v^{-\gamma}$ (at low velocities) with

$\gamma=\frac{2}{3-\beta}$.

In this case we can see that our values of $\beta$ and $\gamma$ are roughly consistent with this model (see Figs. 6 and 7), giving an equivalent value for $s$ of $s \approx 3$. In addition, we note that the fact that we get a single power-law behaviour in this plot mitigates against the arguments put forward in Smith et al. (1997) for the increase in the exponent of $m(v)$. They suggest that the exponent increase results from a change in shape of the bowshock (from $s=2$ to $s=3$ ). However, this could not happen without changing the slope of our $m(t)$ relation.

\subsection{The intensity-velocity relations}

Figures 8 and 9 contain plots of the time-averaged intensityvelocity relations for the $\mathrm{CO} J=2-1$ line, $I_{\mathrm{CO}}(v)$, and for 


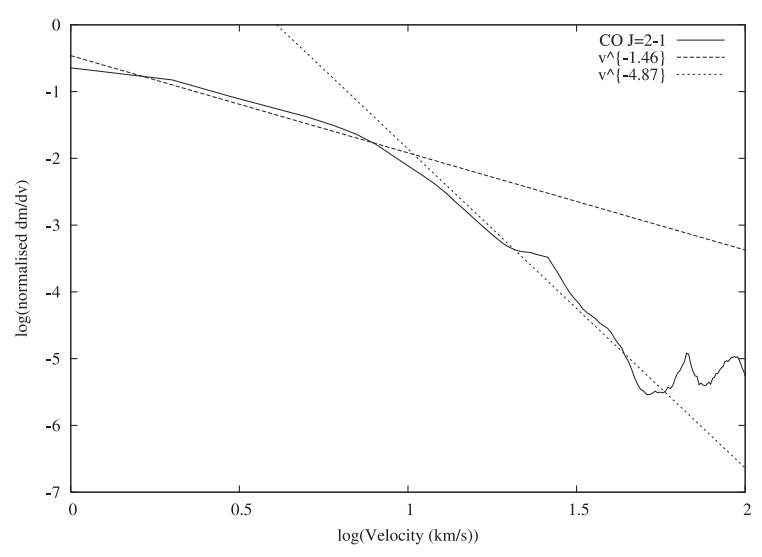

Fig. 8. Plot of the $I_{\mathrm{CO}}(v)$ relation calculated assuming the jet is inclined at $30^{\circ}$ to the plane of the sky, time-averaged over $t=1500 \mathrm{yr}$ to $t=$ $2300 \mathrm{yr}$ and normalised by its total integrated over velocity.

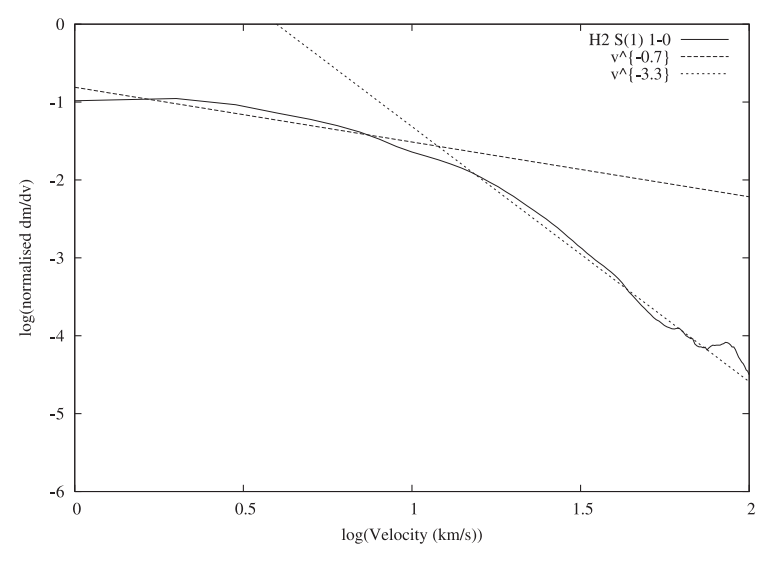

Fig. 9. Plot of the $I_{\mathrm{H}_{2}}(v)$ relation calculated assuming the jet is inclined at $30^{\circ}$ to the plane of the sky, time-averaged over $t=1500 \mathrm{yr}$ to $t=$ $2300 \mathrm{yr}$ and normalised by its total integrated over velocity.

the $\mathrm{H}_{2} \mathrm{~S}(1)$ 1-0 line, $I_{\mathrm{H}_{2}}(v)$, respectively. It is clear from both plots that each of these relations follow an approximate broken power-law, unlike the $m(v)$ relation (due to temperature increasing with higher velocity, see Downes \& Cabrit 2003 for a full explanation of this). This is in agreement with observations.

It is interesting that $I_{\mathrm{H}_{2}}(v)$ is shallower than $I_{\mathrm{CO}}(v)$ at all velocities and times (see Fig. 5). This is due to the $\mathrm{H}_{2}$ line being of higher excitation than the CO line (Downes \& Cabrit 2003). We do not find $I_{\mathrm{H}_{2}}(v)$ to be an increasing function of velocity at low velocities, in contrast to some results of Rosen \& Smith (2004). The reason for this discrepancy is not yet clear, and is being studied currently. In any case, our results for $I_{\mathrm{H}_{2}}(v)$ are qualitatively reasonably close to those actually observed (Salas \& Crúz-González 2002).

The long-duration simulation results here are in good qualitative agreement with those of previous simulations for much shorter time-scales. This indicates that, with the exception of the issue of the increase of $\gamma$ with time, fairly short duration simulations are adequate to capture the main characteristics of jet-driven molecular outflows. It should be noted, however, that this conclusion can only be considered valid for jets which do not vary significantly in direction, and are not episodic.

\subsection{The episodic jet}

As mentioned in Sect. 1, simulations of young jets cannot be used to study the effects of long-period episodicity on jetdriven outflows. In this section we compare our results for the pulsed jet, as described above, with those for the episodic jet (see Sect. 2.3).

\subsubsection{Morphology}

From Fig. 3 we can see that the head of the bowshock of the episodic jet, as expected, does not propagate quite as fast as that of the pulsed jet. This is due to the smaller amount of momentum and energy being injected by the episodic jet when averaged over time resulting from it being virtually "switched off" in between outbursts. However, the length-to-width ratio of the molecular outflow produced by the episodic jet $(\sim 8.1)$ is not significantly smaller than that produced by the pulsed jet $(\sim 10.2)$. In fact, it is quite striking how similar the two outflows are, at least qualitatively.

The lack of a large difference in propagation speeds for the bowshock arises from the material at the head of the bowshock being extremely dense in comparison to that in the ambient medium. This high density arises principally from radiative cooling in the post-shock region. Hence the momentum density in this dense cool shell of material is very high and so, even while the episodic jet is not impacting on the head of the bowshock, the latter does not decelerate quickly.

It is worth while looking at the bowshocks created by the "turning on" of the jet. These bowshocks have been postulated to be a possible origin of very steep $m(v)$ relations in some outflows (Arcé \& Goodman 2001). However, we can see from Fig. 3 that the bowshock from any injection event will not sweep up a significant amount of material as it propagates. This bowshock propagates into the cocoon, which is of very low density having been evacuated by the passage of the main bowshock. When the shock reaches the edge of the cocoon it simply becomes part of the very complex structure of shocks in the ambient material already swept up. It does not alter the morphology of the main bowshock significantly, and neither does it cause the sweep up of more ambient material. The implications of this for the $m(v)$ relation will be discussed more fully in Sect. 3.4.2.

\subsubsection{The mass-velocity relations}

Figure 10 contains plots of the time-averaged $m(v)$ relation for both the pulsed and episodic jets. We can see that, contrary to what would be expected from the arguments of Arcé \& Goodman (2001), the $m(v)$ relation for the episodic jet is no steeper than that for the pulsed jet. From Fig. 3 we can see that the bowshocks created by the episodicity of the jet are, in terms of swept-up mass, extremely weak since they propagate into the cocoon formed by the passage of previous jet material. The material in this cocoon is rather tenuous in comparison to the ambient medium and so one would not expect there to be a qualitative effect of episodicity on the $m(v)$ relation. The arguments of Arcé \& Goodman (2001) would be expected to hold 


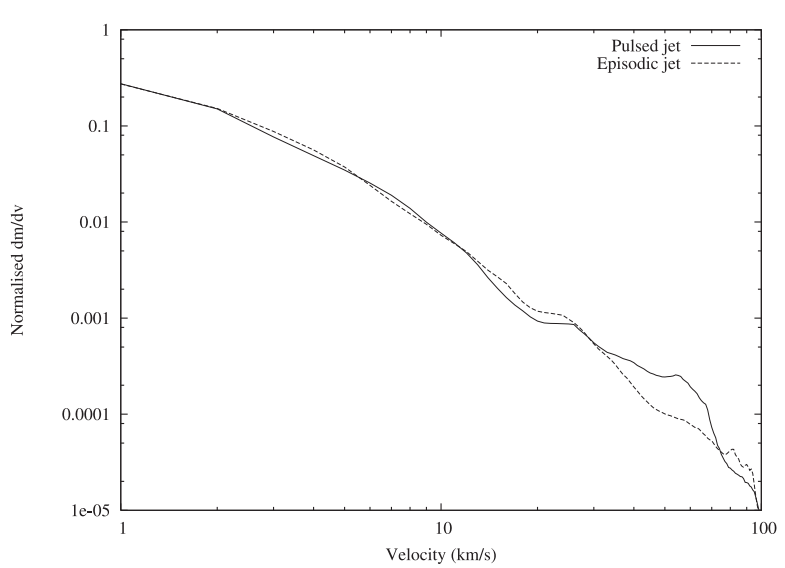

Fig. 10. Plots of the time-averaged total $m(v)$ relations for both the pulsed and the episodic jets, averaged over $t=1500 \mathrm{yr}$ to $t=2300 \mathrm{yr}$.

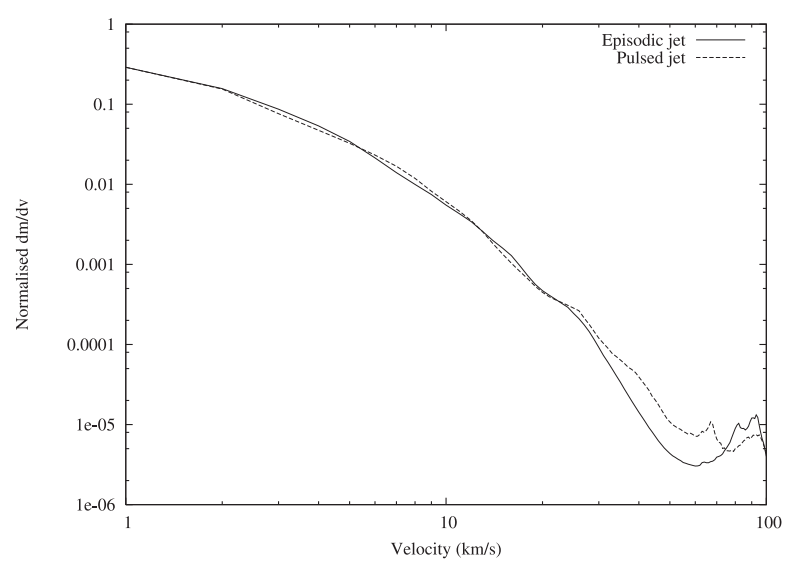

Fig. 11. Plots of the time-averaged $m_{\mathrm{H}_{2}}(v)$ relations for both the pulsed and the episodic jets, averaged over $t=1500 \mathrm{yr}$ to $t=2300 \mathrm{yr}$.

if there were some process which injected high density material into the cocoon such as, perhaps, strong turbulence in the ambient medium.

Figure 11 contains plots of the $m_{\mathrm{H}_{2}}(v)$ relations for both the pulsed and episodic jets. Again, we can see that, certainly at low velocities $\left(v \leq 30 \mathrm{~km} \mathrm{~s}^{-1}\right)$, there is no significant difference between the two. At higher velocities there is a slight depletion, by a factor of $\sim 2$, of $\mathrm{H}_{2}$ in the episodic jet when compared with the pulsed jet. Detailed examination of the temporal behaviour of the $m_{\mathrm{H}_{2}}(v)$ relations for each of the simulations strongly indicates that the differences at these velocities are, in fact, the result of the large-scale corrugation in the bowshock of the pulsed jet simulation roughly half-way along the length of the jet (see Fig. 3). Thus this discrepancy in the results of the simulations is more due to the detail of the structure of the bowshock rather than the fact that one of the jets is episodic in nature.

\subsubsection{The intensity-velocity relations}

Figure 12 contains plots of the time-averaged $I_{\mathrm{CO}}(v)$ relations for each of the pulsed and episodic jets. It is clear that, at least below $v \sim 20 \mathrm{~km} \mathrm{~s}^{-1}$, the relations do not differ much. This is due to the similarities of the morphologies of the pulsed and

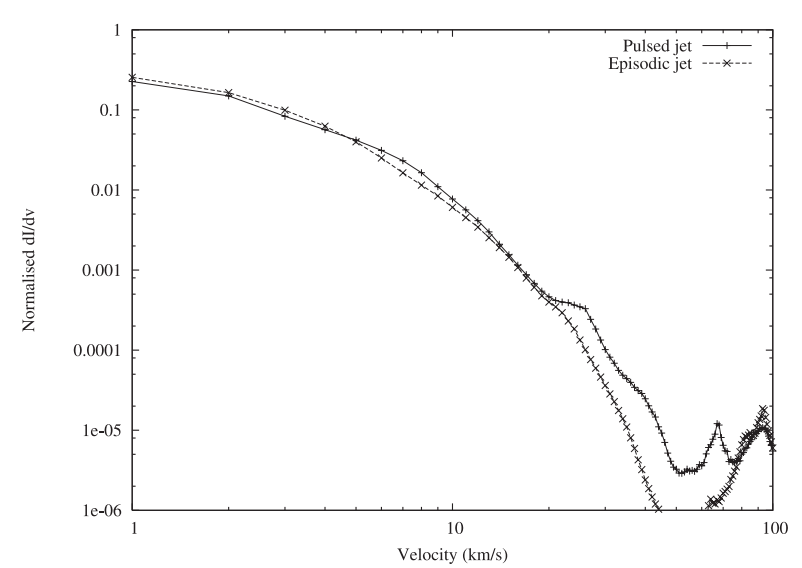

Fig. 12. Plots of the time-averaged $I_{\mathrm{CO}}(v)$ relations for both the pulsed and the episodic jets, averaged over $t=1500 \mathrm{yr}$ to $t=2300 \mathrm{yr}$.

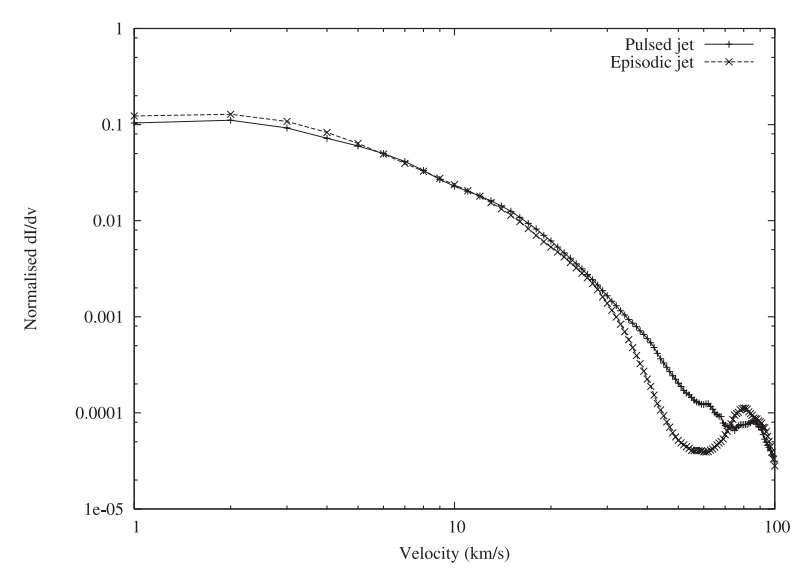

Fig. 13. Plots of the time-averaged $I_{\mathrm{H}_{2}}(v)$ relations for both the pulsed and the episodic jets, averaged over $t=1500 \mathrm{yr}$ to $t=2300 \mathrm{yr}$.

episodic jets, which is also expressed in their $m(v)$ relations (see Sect. 3.4.2). Above $20 \mathrm{~km} \mathrm{~s}^{-1}$, however, the $I_{\mathrm{CO}}(v)$ for the episodic jet can be seen to be somewhat steeper resulting in a maximum ratio between the two plots of $\sim 7.5$ at $55 \mathrm{~km} \mathrm{~s}^{-1}$.

These differences are probably not observable in real systems, occurring, as they do, at such high velocities and low intensities. As in Sect. 3.4.2, these differences arise from slight differences in the bowshock, rather than directly from the presence of episodicity in one of the simulations.

Finally, Fig. 13 contains plots of the $I_{\mathrm{H}_{2}}(v)$ relations. The picture here is very similar to that for the $I_{\mathrm{CO}}(v)$ relations, and can be explained in the same way.

Overall, then, we do not find any evidence to suggest that long-period episodicity in the driving jet will change the observed characteristics, certainly in the intensity-velocity relations, of a molecular outflow.

\section{Conclusions}

We have run the first simulations of jet-driven molecular outflows up to the age of $2300 \mathrm{yr}$, a significant fraction of the dynamical age of at least some molecular outflows. We have presented mass- and intensity-velocity relations for both 
a simulation of a pulsed jet and a jet displaying long-period (800 yr) episodicity. Our main results are as follows:

- The exponents in the mass- and intensity-velocity relations, $\gamma$, at low velocities do increase with time, up to about $t \sim 1500 \mathrm{yr}$ after which it reaches an approximate steady state. Although this is not certain from our simulations, detailed study leads us to believe with some confidence that $\gamma$, while it may vary somewhat, will not have a systematic increase after this. The final values for the exponents are $\sim 1.6, \sim 0.7$ and $\sim 1.78$ for the $m(v), I_{\mathrm{H}_{2}}(v)$ and $I_{\mathrm{CO}}(v)$ relations respectively, in good agreement with observations.

- The presence of long-period episodicity does not significantly alter either the mass- or intensity-velocity relations. This is due to the low density of the cocoon around the jet into which the bowshocks arising from the episodicity propagate.

- There continue to be large variations in each of the massand intensity-velocity relations at high velocities due to fluid instabilities at the head of the bowshock.

- The model of Downes \& Cabrit (2003) appears to be successful in modeling even the results of long-duration simulations.

\subsection{Caveats and future work}

It is reasonable to ask what the effect of the assumption of cylindrical symmetry is. We believe that it may have an impact at high velocities, where large variations in the $m(v)$ and $I(v)$ relations occur. These variations arise from instabilities at the bowshock resulting in corrugations being "shed" from the head of the bowshock. In cylindrical symmetry these shedding events will be of larger scale and more coherent than would be expected in a full 3-D simulation. Hence one would expect that the high-velocity variations in $m(v)$ and $I(v)$, while still present, would be smaller in amplitude in a full 3-D system.

It is also worth noting that in these simulations we have used a homogeneous ambient medium while, on length scales as large as the ones simulated here, it could reasonably be expected that the ambient medium would not be uniform. While a detailed discussion of the effect of an inhomogeneous ambient medium is beyond the scope of this paper, we make the following remarks.

If the ambient density were to fall off with distance from the source one might expect that the $m(v)$ relation will become steeper, since the fastest material is that at the head of the bowshock, and here the density (and hence amount of mass) drops with time. However, this reasoning does not take into account the widening of the bowshock which would result from passing through such a stratified medium. A wider bowshock tends to produce shallower $m(v)$ relations (Downes \& Cabrit 2003) and this effect may cancel out the steepening of the $m(v)$ relation expected from a decreasing ambient density. Similar arguments hold if the density increases with distance from the source.

In addition, it is possible that such a long jet would encounter random "clumps" of high density material. It is difficult to predict what effect this would have on the $m(v)$ relation, although clumps with a typical size smaller than the jet radius are unlikely to influence such a global property significantly. In short, it is not clear what effect, if any, variations in the ambient density will have on the $m(v)$ relation. These issues will be the focus of future work.

Finally, dynamically significant magnetic fields can affect the expected $m(v)$ relations since they will change the bowshock morphology. The precise nature of these changes will depend on the magnetic field topology. For example, if there were a significant global toroidal field, or even one aligned to the jet axis, the bowshock would be narrower and hence we would expect a steeper $m(v)$ relation (Downes \& Cabrit 2003). However, a magnetic field perpendicular to the jet axis would tend to lead to a broader bowshock and hence a shallower $m(v)$ relation.

Acknowledgements. We wish to thank S. Cabrit for useful comments during the writing of this paper. This work was partially funded by the Enterprise Ireland Basic Research Grant Scheme, grant number SC/1999/268/Y and also by the the CosmoGrid project, funded under the Programme for Research in Third Level Institutions (PRTLI) administered by the Irish Higher Education Authority under the National Development Plan and with partial support from the European Regional Development Fund.

\section{References}

Arcé, H. G., \& Goodman, A. A. 2001, ApJ, 551, L171

Bachiller, R., \& Tafalla, M. 1999, in The Origin of Stars and Planetary Systems, ed. C. J. Lada, \& N. D. Kylafis (Kluwer Academic Publishers), 227

Bence, S. J., Richer, J. S., \& Padman, R. 1996, MNRAS, 279, 866

Blondin, J. M., Fryxell, B. A., \& Königl, A. 1990, ApJ, 360, 370

Dgani, R., van Buren, D., \& Noriega-Crespo, A. 1996, ApJ, 461, 927

Dove, J. E., \& Mandy, M. E. 1986, ApJ, 311, L93

Downes, T. P., \& Cabrit, S. 2003, A\&A, 403, 135

Downes, T. P., \& Ray, T. P. 1998, A\&A, 331, 1130

Downes, T. P., \& Ray, T. P. 1999, A\&A, 345, 977

Falle, S. A. E. G., \& Raga, A. C. 1995, MNRAS, 272, 785

Lee, C.-F., Mundy, L. G., Reipurth, B., Ostriker, E. C., \& Stone, J. M. 2000, ApJ, 542, 925

Lepp, S., \& Shull, M. J. 1983, ApJ, 270, 578

Lim, A. J., Raga, A. C., Rawlings, J. M. C., \& Williams, D. A. 2002, MNRAS, 335, 817

Noriega-Crespo, A., Garnavich, P. M., Raga, A. C., Canto, J., \& Bohm, K.-H. 1995, BAAS, 27, 1318

Raga, A. C., \& Cabrit, S. 1993, A\&A, 278, 267

Richer, J. S., Shepherd, D. S., Cabrit, S., Bachiller, R., \& Churchwell, E. 2000, in Protostars and Planets IV, ed. V. Mannings, A. P. Boss, \& S. S. Russell (University of Arizona Press), 867

Rodríguez, L. F., Carral, P., Moran, J. M., \& Ho, P. T. P. 1982, ApJ, 260, 635

Rosen, A., \& Smith, M. D. 2004, MNRAS, 347, 1097

Rossi, P., Bodo, G., Massaglia, S., \& Ferrari, A. 1997, A\&A, 321, 672

Salas, L., \& Crúz-González, I. 2002, ApJ, 572, 227

Smith, M. D., Suttner, G., \& Yorke, H. W. 1997, A\&A, 323, 223

Stahler, S. 1994, ApJ, 422, 616

Sutherland, R. S., \& Dopita, M. A. 1993, ApJS, 88, 253

van Leer, B. 1977, J. Comp. Phys., 23, 276

Zhang, Q., \& Zheng, X. 1997, ApJ, 474, 719 